\title{
Small-bowel obstruction due to excessive consumption of oleaster-leafed pear (Pyrus elaeagnifolia Pall.): A retrospective study
}

\author{
Serkan KARAISLI ${ }^{1}$ and Fatih Karayol ${ }^{2}$ \\ ${ }^{1}$ Izmir Katip Celebi University Faculty of Medicine \\ ${ }^{2}$ Mus State Hospital
}

April 27, 2021

\begin{abstract}
Background: Small-bowel obstruction (SBO) is a common emergency worldwide and adhesions are the main cause. Oleasterleafed pear (Pyrus elaeagnifolia Pall.) is an endemic wild pear species in Anatolia. The wild pear fruits are consumed for the treatment of diarrhea in traditional medicine in Turkey. Here, we aimed to describe SBO caused by excessive consumption of wild pear and compare clinical features of it with those in adhesive SBO. Methods: The medical records of patients who were followed with a diagnosis of SBO between May 2018 and September 2019 were reviewed. Patients with wild pear-related SBO (Group 1) and patients with adhesive SBO (Group 2) were included in the study. Patient characteristics, blood parameters and clinical features were compared. Results: A total of 74 patients including 16 in Group 1 and 58 in group 2 were included. Amount of wild pear consumed was median 600 grammes (range, 450-800 gr). The median age in Group 1 and Group 2 was 55 and 60.5 , respectively. History of previous SBO was more common in Group $2(\mathrm{p}=0.032)$. Increased levels of WBC, CRP and BUN were significantly more frequent in Group 2 (all $\mathrm{p}<0.05$ ). Group 1 was associated with a shorter duration of complaints and faster recovery (all $\mathrm{p}<0.05)$. Surgical intervention was required for six patients $(10.3 \%)$ in only Group 2 . Conclusion: The potential adverse effects of over-consumed traditional medicines should be kept in mind. Questioning the last food consumed before the complaints start may be a clue for food-induced SBO.
\end{abstract} Small-bowel obstruction due to excessive consumption of oleaster-leafed pear (Pyrus elaeag-
nifolia Pall.): A retrospective study

\section{Abstract}

Background: Small-bowel obstruction (SBO) is a common emergency worldwide and adhesions are the main cause. Oleaster-leafed pear (Pyrus elaeagnifolia Pall. ) is an endemic wild pear species in Anatolia. The wild pear fruits are consumed for the treatment of diarrhea in traditional medicine in Turkey. Here, we aimed to describe SBO caused by excessive consumption of wild pear and compare clinical features of it with those in adhesive SBO.

Methods: The medical records of patients who were followed with a diagnosis of SBO between May 2018 and September 2019 were reviewed. Patients with wild pear-related SBO (Group 1) and patients with adhesive SBO (Group 2) were included in the study. Patient characteristics, blood parameters and clinical features were compared.

Results: A total of 74 patients including 16 in Group 1 and 58 in group 2 were included. Amount of wild pear consumed was median 600 grammes (range, 450-800 gr). The median age in Group 1 and Group 2 was 55 and 60.5 , respectively. History of previous SBO was more common in Group $2(\mathrm{p}=0.032)$. Increased levels of WBC, CRP and BUN were significantly more frequent in Group 2 (all $\mathrm{p}<0.05$ ). Group 1 was associated 
with a shorter duration of complaints and faster recovery (all $\mathrm{p}<0.05$ ). Surgical intervention was required for six patients (10.3\%) in only Group 2.

Conclusion: The potential adverse effects of over-consumed traditional medicines should be kept in mind. Questioning the last food consumed before the complaints start may be a clue for food-induced SBO.

Keywords: adhesive bowel obstruction; oleaster-leafed pear; small-bowel obstruction; traditional medicine; wild fruit.

\section{WHAT'S KNOWN?}

* Small-bowel obstruction is one of the most common causes of emergency service admission.

*Fruit-related phytobezoars are the most common reason for food-related small bowel obstruction.

\section{WHAT'S NEW?}

*Wild fruits which are consumed for traditional medicine popular may cause small bowel obstruction.

*Questioning 'the last food' consumed just before the complaints start may give a clue about food-related small bowel obstruction.

*This study is the first which report a series of patients with unfamiliar bowel obstruction associated with the wild fruit.

*This study is the first series describing food-induced SBO which obstruction mechanism is not phytobezoars.

\section{Introduction}

Small-bowel obstruction (SBO) is one of the most common causes of emergency service admission. Prior abdominal surgery-related adhesions are the cause of $65-75 \%$ of cases. Hernias, neoplasms, Crohn's disease, bezoars, foreign bodies and intussusceptions are other etiological causes of SBO. ${ }^{1,2}$ Food-related SBO was described for many fruits including citrus fruit, persimmons, orange pith, mango, carrots, Brussels sprouts, wild banana and dried fruits. However, all those reports described phytobezoars as the mechanism of obstruction. ${ }^{3-5}$

Pear (Pyrus) is one of the most bred and consumed fruits in the world. There are hundreds of Pyrus species but only 23 wild species were described, all native to Europe, northern Africa and temperate regions of Asia. ${ }^{6}$ Oleaster-leafed pear (Pyrus elaeagnifolia Pall.) is one of the endemic wild pear species of Anatolia. Besides Turkey, it is grown also in south Ukraine, Albania, Bulgaria, Greece and Romania. ${ }^{6}$ The wild pear fruits mostly consumed by local people and also preferred especially for the treatment of diarrhea in traditional medicine in Turkey. ${ }^{7}$

In our city, this oleaster-leafed pear tree grows naturally and fruits are frequently consumed by local people during the harvest season. It was noticed that many patients who excessively consumed this wild pear for traditional diarrhea treatment admitted to the emergency department with a SBO presentation during the harvest season. In the present study, we aimed to describe this unfamiliar food-related SBO entity and compare clinical characteristics of it with those in adhesive SBO.

\section{Material and Methods}

The medical records of adult patients ( $>18$ years old) who hospitalized due to SBO in Mus State Hospital, which is a rural hospital in East Turkey, between May 2018 and September 2019 were collected prospectively and reviewed retrospectively. Patient data were collected from the patient files and hospital software database.

Patients with SBO due to excessive consumption of oleaster-leafed pear(Figure 1) and patients with adhesive SBO were included in the study. Patients with an incarcerated hernia, internal hernia, primary malignant obstruction, bezoars, gallstone ileus, drug / hypokalemia-related paralytic ileus, volvulus, intussusception, any colonic obstruction, and early ([?] 6 weeks from index abdominal operation) postoperative 
obstruction were excluded. Patients with haematological disorders, chronic renal failure, urinary system disease, congestive heart failure, active cancer, and those with incomplete records were also excluded from the study. After the initial evaluation, 74 patients who met the study criteria were included. Patients were divided into two groups as SBO due to excessive consumption of wild pear (Group 1), and adhesive SBO (Group 2).

Patient characteristics such as age, sex, comorbid diseases, number of previous abdominal operations and type of incisions, history of previous intestinal obstruction; and clinical features including duration of complaints, duration of hospital stay, duration of nasogastric tube drainage, time of enteral feeding, duration of radiological recovery (time until the disappearance of air-fluid levels on erect plain radiograph), operations and presence of recurrence during the follow-up were reviewed. In addition, blood parameters including white blood cell (WBC) (reference range: 4500-10000/ $\mu \mathrm{L}$ ), C-reactive protein (CRP) (reference range: 0-5 mg/dL), blood urea nitrogen (BUN) (reference range: $8-20 \mathrm{mg} / \mathrm{dL}$ ), creatinine (reference range: $0.6-1.2 \mathrm{mg} / \mathrm{dL}$ for men and $0.5-1.1 \mathrm{mg} / \mathrm{dL}$ for women), BUN/creatinine ratio (reference range: 10-20) and potassium values (reference range: $3.5-5.1 \mathrm{mmol} / \mathrm{L}$ ) were noted at the day of admission to the emergency department in all patients. Approximate amount of wild pear which patients in Group 1 consumed was also recorded at the first admission. Patient characteristics and study parameters were compared between the two groups.

All patients were evaluated in emergency service. Diagnosis of bowel obstruction was established according to history, physical examination, laboratory and radiological examination (Figure 2) of the patients. The last meal/food before the complaints started were questioned. In suspicion of bowel obstruction, a nasogastric tube and urinary catheter were inserted and an enema was applied. Enteral feeding was stopped and the intravenous fluid was administered. Patients whose clinical evaluation was compatible with a complete obstruction underwent emergency surgery. When partial bowel obstruction were determined, patients were hospitalized for further examination and management. Patients with a partial obstruction were administered intravenous fluid and enema, and oral intake was stopped. Daily blood parameters and plain abdominal radiographs were performed. If clinical improvement was obtained, nasogastric and urinary catheters were removed and oral intake was started. If there was no response to conservative treatment in the first 72 hours or peritoneal irritation signs appeared in any time, surgical intervention was decided. Open surgery was the preferred approach due to lack of experience in laparoscopic adhesiolysis. The colonoscopic examination was performed to exclude colonic obstruction if a patient has not undergone colonoscopy in the last one year. Follow-up of the patients continued until May 2020.

Written permission for this study was obtained from the administration of the Mus State Hospital (Date: 04/30/2020, Decision No: 7407). Ethics committee approval was obtained from the Non-Interventional Clinical Studies Institutionel Review Board of Izmir Kâtip Celebi University (Decision number: 877, Decision date: 09.17.2020) due to the absence of a local ethics committee in Mus city. Written informed consents were obtained from all patients. The study was performed in accordance with the ethical standards as laid down in the 1964 Declaration of Helsinki.

Statistical analysis

Statistical Package for Social Sciences version 22.0 (IBM Corp.; Armonk, NY, USA) was used for all statistical analyses. Variables were reflected as the mean \pm standard deviation and frequency values. The Levene test was performed for the homogeneity of variances. The assumption of normality was done with the ShapiroWilk test. Student's t-test was used for continuous variables if the parametric test meets the prerequisites, otherwise, Mann Whitney-U test was performed. Categorical variables were compared by Chi-square test or Fisher's exact test. $\mathrm{p}<0.05$ was considered as statistically significant.

\section{Results}

A total of 74 patients including 16 in Group 1 and 58 in group 2 were included in the study. Amount of wild pear which patients in Group 1 consumed was median 600 grammes (range, 450-800 gr). All patients in Group 1 were admitted to the hospital between the end of August and the end of November and that period was consistent with the harvesting period of wild pears. 
The median age in Group 1 and Group 2 was 55 (range, 23-74) and 60.5 (range, 18-93), respectively. Male to female ratio was 7 in Group 1 and it was statistically significantly higher compared to Group 2 ( $\mathrm{p}=0.01)$. Comorbid diseases were more common in Group 2 and hypertension $(\mathrm{n}=15)$ and diabetes mellitus $(\mathrm{n}=10)$ were the most seen comorbidities in study group. Age, comorbidities and history of previous operation were comparable between groups. History of previous SBO was significantly higher in Group $2(\mathrm{p}=0.032)$. Patients in Group 2 had significantly abnormal levels of WBC, CRP and BUN compared to Group 1 (all p $<0.05$ ), however, there was no difference in levels of creatinine, potassium and BUN/creatinine ratio between groups (all $\mathrm{p}>0.05$ ). Group 1 was associated with a shorter duration of complaints and shorter length of hospital stay and also faster clinical recovery (all $\mathrm{p}<0.05)$. Surgical intervention was required for six patients $(10.3 \%)$ in Group 2. Four patients (6.9\%) underwent surgery on the fourth day of admission due to the absence of clinical improvement. Other two (3.4\%) patients developed an acute abdomen in the second day of admission and taken into exploratory laparotomy. Adhesiolysis in four (6.9\%), and segmental intestinal resection and anastomosis were performed in two patients (3.4\%). Intestinal resection and anastomosis required only in those who developed an acute abdomen. Postoperative superficial surgical site infection and urinary tract infection were developed in two patients $(3.4 \%)$ and in one patient (1.7\%), respectively. Although the mean follow-up period was significantly longer in Group 1 compared to Group 2, no recurrence occurred. In group 2 , SBO was relapsed in none of the patients who underwent laparotomy. However, 11 patients $(21.2 \%)$ among 52 patients conservatively managed readmitted to the hospital due to recurrence of SBO during a median of 11-month (range, 7-22 months) follow-up. All patients were treated conservatively again. The demographic characteristics and clinicopathologic features of patients are summarized in Table 1 .

\section{Discussion}

The clinical presentations and severity of SBO depend on the level of the blockage. Nausea, vomiting, abdominal cramps and distention, and the decrease or absence of the passage of stool and flatus are the main clinical manifestations of SBO. The more proximally obstruction site is located, the more fastly symptoms ocur. $^{8}$ For the prediction of an adhesive SBO, history of a previous abdominal surgery has a sensitivity and specificity of $85 \%$ and $78 \%$, respectively. ${ }^{9}$ In the present study, two patients $(2.7 \%)$ in Group 2 denied any medical history for a possible cause of adhesion including abdominal operation, peritonitis, endometriosis or acute diverticulitis. On radiological examination, the level of obstruction was the terminal ileum in these two patients, while other patients had different levels of blockage which was possibly the adhesion site.

Blood markers are specific in nor in the diagnosis of SBO neither in determining the severity of the disease. In a prospective study of Cosse et al., ${ }^{10}$ procalcitonin with a value of above $0.57 \mathrm{ng} / \mathrm{mL}$ was found to be a potential predictor for small bowel ischemia with a sensitivity of $83 \%$. Radiological methods including erect/lateral decubitus plain radiographs, ultrasound (US) and computed tomography (CT) are applicable to establish a diagnosis. ${ }^{8}, 9$ A plain radiograph is considered as the first step radiological study. It is a diagnostic tool in $50-60 \%$ of the patients, while inconclusive or misleading for others. ${ }^{1,8,11}$ The US is a reliable tool with $90 \%$ sensitivity in the diagnosis of SBO. The US can reveal the presence of $>2.5$-cm dilated small bowel loops and decreased bowel peristalsis. ${ }^{1,9}$ Diagnostic value of intravenous contrast-enhanced CT is superior to plain radiograph and US. In addition to this, CT enables to determine the severity and location of the obstruction, possible cause for obstruction, and also to detect potential complications such as bowel ischemia and perforation. ${ }^{1,8}$ Oral contrast is considered not to be required in the diagnosis of SBO. On the other hand, oral contrast used during conservative management is associated with less requirement of laparotomy and shorter hospital stay. ${ }^{1,12}$ As our daily practice, we performed an erect plain radiograph as the first step imaging for suspected cases. In the presence of air-fluid levels, an abdominal CT was used in most patients in adhesive SBO group to identify the level and severity of obstruction, and also eliminate other possible abdominal pathologies. Due to its having onset in the last 24 hours and the absence of description in the available literature, a routine CT was performed for all patients in the wild pear group to understand the mechanism of obstruction (partial/total) (paralytic/mechanic).

Conservative treatment is considered as the preferred treatment option for adhesive SBO in the absence of acute abdomen signs or if there is no evidence of intestinal ischemia or perforation. Approximately three out 
of four SBO patients can be managed conservatively. ${ }^{8}$ The optimal duration of conservative treatment is still unclear. However, 72 hours is thought a critical cutoff to review the operative option since a failed conservative treatment exceeding 72 hours is correlated with increased risk of small bowel resection, longer hospital stay and higher morbidity. ${ }^{1,13-15}$ Surgical intervention is mandatory in case of small bowel ischemia/perforation or failure of conservative treatment. Historically, laparotomic adhesiolysis has been the preferred approach for adhesive SBO. ${ }^{1,2}$ Laparoscopic adhesiolysis had been described in recent decades, and it was associated with a reduced risk of morbidity and in-hospital mortality. ${ }^{15,}{ }^{16}$ Recurrence of SBO occurs in $12 \%$ of conservatively treated patients within 1 year and $20 \%$ of them after 5 years. That risk is $8 \%$ within 1 year and $16 \%$ after 5 years after surgical treatment. ${ }^{17}$ In the adhesive SBO group, 6 (10.3\%) patients underwent surgery. Interestingly, all patients in the wild pear group dramatically had clinical improvement on the first day of admission. Recurrence only occurred after conservative treatment in the adhesive SBO group. Recognition of excessive consumption of wild pear as a predisposing factor for SBO and increased patient conscious during hospital stay possibly played a role in preventing recurrence in the wild pear group.

Patients in the wild pear group were considered as adhesive SBO at admission due to their frequently having a history of previous abdominal operation. Management of treatment was planned similar to adhesive SBO. The mechanism of obstruction was not clear after excessive consumption of wild pear. We evaluated patients for possible causes of obstruction. 1) At first, phytobezoar seemed to be a possible cause of obstruction due to being food-related SBO and declaration of consuming pear seeds by a few patients. However, all patients had a partial SBO and CT revealed the absence of a phytobezoar in the level of obstruction. 2) Recognition of hyperactive bowel sounds at admission eliminated the possibility of paralytic obstruction. 3) Hypokalemia-related pseudo-obstruction was ruled out because serum potassium levels were within or above normal limits.

On the other hand, some other possible mechanisms we could not identify might be playing a role. Oleasterleafed pear might be including an unidentified metabolite (or metabolites) which inhibiting gastrointestinal motility by using several pathways. Psyllium is a well-known diet fibre source and widely used against both constipation and diarrhea ${ }^{18}$ Mehmood et al. ${ }^{18}$ reported a possible mechanism of the antidiarrheal effect of psyllium husk. They thought blockage of $\mathrm{Ca}^{+2}$ channels and activation of nitric oxide/cyclic guanosine monophosphate pathways by undefined components might be inhibiting gastrointestinal motility.

Traditional medicine is still preferred especially in rural regions and countries with inadequate health service. Endemic trees and herbs constitute the majority of medicines. Although oleaster-leafed pear grows in a limited region including Anatolia and Balkans, 23 different wild pear species grow in different regions worldwide. ${ }^{6}$ Other wild pear species or fruits may have a similar impact on the gastrointestinal system. We could not find similar studies in the available literature. All previous studies and case reports have described phytobezoars. Food-related SBO maybe has been neglected or unreported entity.

There are some limitations of the present study. Firstly, this is a retrospective study conducted in a singlecenter. Additionally, the described wild pear type grows in a limited area in the world and these results may not be applicable to other pear species. Finally, the number of patients is inadequate to draw strong conclusions. On the other hand, the main strength of the present study is it's being the first series describing food-induced SBO which obstruction mechanism is not phytobezoars.

\section{Conclusion}

The dose of the traditional medicines can not be regulated and exaggerated treatment may mimic some clinical conditions. Physicians should be aware of different manifestations of familiar diseases, such as SBO, which may occur following endemic fruit consumption, especially in the regions where traditional medicine popular. Questioning 'the last food' consumed just before the complaints start may give a clue about foodrelated SBO. Recognizing typical symptoms of excessive consumption of any food may contribute physicians to plan the optimal management and avoid unnecessary surgical interventions.

Acknowledgments : The authors thank Kasım Coşkun and Bilal Tekin for their procuring photos of the tree and fruit. There are no conflicts of interest in connection with this paper. There is no financial disclosure 
in connection with this paper.

\section{References}

1. Catena F, De Simone B, Coccolini F, Di Saverio S, Sartelli M, Ansaloni L. Bowel obstruction: a narrative review for all physicians. World J Emerg Surg. 2019;14:20. doi: 10.1186/s13017-019-0240-7.

2. ten Broek RP, Issa Y, van Santbrink EJ, Bouvy ND, Kruitwagen RF, Jeekel J et al. Burden of adhesions in abdominal and pelvic surgery: systematic review and met-analysis. BMJ. 2013;347:f5588. doi: 10.1136/bmj.f5588.

3. Lohn JW, Austin RC, Winslet MC. Unusual causes of small-bowel obstruction. J R Soc Med. 2000;93(7):365-368. doi: 10.1177/014107680009300707.

4. Teng TZJ, Tan YP, Shelat VG. Persimmon fruit causing simultaneous small bowel and stomach obstruction. Singapore Med J. 2019;60(10):550. doi: 10.11622/smedj.2019132.

5. Slesak G, Mounlaphome K, Inthalad S, Phoutsavath O, Mayxay M, Newton PN. Bowel obstruction from wild bananas: a neglected health problem in Laos. Trop Doct. 2011;41(2):85-90. doi: 10.1258/td.2011.100293.

6. Silva GJ, Souza TM, Barbieri RL, Oliveira ACD. Origin, domestication, and dispersing of pear (Pyrus spp.). Adv Agr. 2014;2014:e541097. doi: 10.1155/2014/541097

7. Baytop T. Therapy with Medicinal Plants in Turkey, Past and Present. 2nd ed. Istanbul: Nobel; 1999.

8. Vilz TO, Stoffels B, Strassburg C, Schild HH, Kalff JC. Ileus in Adults. Dtsch Arztebl Int. 2017;114(2930):508-518. doi: 10.3238/arztebl.2017.0508.

9. Taylor MR, Lalani N. Adult small bowel obstruction. Acad Emerg Med. 2013;20(6):528-544. doi: 10.1111/acem.12150.

10. Cosse C, Regimbeau JM, Fuks D, Mauvais F, Scotte M. Serum procalcitonin for predicting the failure of conservative management and the need for bowel resection in patients with small bowel obstruction. J Am Coll Surg. 2013;216(5):997-1004. doi: 10.1016/j.jamcollsurg.2012.12.051. Epub 2013 Mar 19.

11. Gans SL, Stoker J, Boermeester MA. Plain abdominal radiography in acute abdominal pain; past, present, and future. Int J Gen Med. 2012;5:525-533. doi: 10.2147/IJGM.S17410. Epub 2012 Jun 13.

12. Branco BC, Barmparas G, Schnüriger B, Inaba K, Chan LS, Demetriades D. Systematic review and meta-analysis of the diagnostic and therapeutic role of water-soluble contrast agent in adhesive small bowel obstruction. Br J Surg. 2010;97(4):470-478. doi: 10.1002/bjs.7019.

13. Schraufnagel D, Rajaee S, Millham FH. How many sunsets? Timing of surgery in adhesive small bowel obstruction: a study of the Nationwide Inpatient Sample. J Trauma Acute Care Surg. 2013;74(1):181-187; discussion 187-189. doi: 10.1097/TA.0b013e31827891a1.

14. Keenan JE, Turley RS, McCoy CC, Migaly J, Shapiro ML, Scarborough JE. Trials of nonoperative management exceeding 3 days are associated with increased morbidity in patients undergoing surgery for uncomplicated adhesive small bowel obstruction. J Trauma Acute Care Surg. 2014;76(6):1367-1372. doi: 10.1097/TA.000000000000246.

15. Ten Broek RPG, Krielen P, Di Saverio S, Coccolini F, Biff WL, Ansaloni L et al. Bologna guidelines for diagnosis and management of adhesive small bowel obstruction (ASBO): 2017 update of the evidencebased guidelines from the world society of emergency surgery ASBO working group. World J Emerg Surg. 2018;13:24. doi: 10.1186/s13017-018-0185-2.

16. Sajid MS, Khawaja AH, Sains P, Singh KK, Baig MK. A systematic review comparing laparoscopic vs open adhesiolysis in patients with adhesional small bowel obstruction. Am J Surg. 2016;212(1):138-150. doi: 10.1016/j.amjsurg.2016.01.030. Epub 2016 Apr 13. 
17. Foster NM, McGory ML, Zingmond DS, Ko CY. Small bowel obstruction: a population-based appraisal. J Am Coll Surg. 2006;203(2):170-176. doi: 10.1016/j.jamcollsurg.2006.04.020. Epub 2006 Jul 7.

18. Mehmood MH, Aziz N, Ghayur MN, Gilani AH. Pharmacological basis for the medicinal use of psyllium husk (Ispaghula) in constipation and diarrhea. Dig Dis Sci. 2011;56(5):1460-71. doi: 10.1007/s10620-0101466-0. Epub 2010 Nov 17.

\section{Table(s)}

Table 1. The demographic characteristics and clinicopathologic features of patients.

\begin{tabular}{|c|c|c|c|}
\hline & & Group $1(\mathrm{n}=16)$ (Wild pear) & Group $2(\mathrm{n}=58)$ (Adhes \\
\hline Age (years) & & $52.6 \pm 14.6$ & $55.9 \pm 18.4$ \\
\hline \multicolumn{4}{|l|}{ Sex } \\
\hline & Female & $2(12.5 \%)$ & $28(48.3 \%)$ \\
\hline & Male & $14(87.5 \%)$ & $30(51.7 \%)$ \\
\hline \multicolumn{4}{|l|}{ Comorbid disease } \\
\hline & Yes & $3(18.8 \%)$ & $23(39.7 \%)$ \\
\hline & No & $13(81.3 \%)$ & $35(60.3 \%)$ \\
\hline Number of previous abdominal operations & & $1.1 \pm 0.6$ & $1.3 \pm 0.6$ \\
\hline & Upper abdominal & $5(31.3 \%)$ & $12(20.7 \%)$ \\
\hline & Lower abdominal & $2(12.5 \%)$ & $24(41.4 \%)$ \\
\hline & Combined & $7(43.8 \%)$ & $22(37.9 \%)$ \\
\hline & No operation & $2(12.5 \%)$ & 0 \\
\hline \multicolumn{4}{|l|}{ History of previous SBO } \\
\hline & Yes & $2(12.5 \%)$ & $24(41.4 \%)$ \\
\hline & No & $14(87.5 \%)$ & $34(58.6 \%)$ \\
\hline \multicolumn{4}{|l|}{ WBC count } \\
\hline & Normal & $14(87.5 \%)$ & $14(24.1 \%)$ \\
\hline & High & $2(12.5 \%)$ & $44(75.9 \%)$ \\
\hline \multicolumn{4}{|l|}{ CRP } \\
\hline & Normal & $8(50 \%)$ & $11(19 \%)$ \\
\hline & High & $8(50 \%)$ & $47(81 \%)$ \\
\hline \multicolumn{4}{|l|}{ BUN } \\
\hline & Normal & $15(93.8 \%)$ & $36(62.1 \%)$ \\
\hline & High & $1(6.3 \%)$ & $22(37.9 \%)$ \\
\hline \multicolumn{4}{|l|}{ Creatinine } \\
\hline & Normal & $15(93.8 \%)$ & $45(77.6 \%)$ \\
\hline & High & $1(6.3 \%)$ & $13(22.4 \%)$ \\
\hline \multicolumn{4}{|l|}{$\mathrm{BUN} /$ creatinine ratio } \\
\hline & Normal & $8(50 \%)$ & $36(62.1 \%)$ \\
\hline & High & $8(50 \%)$ & $22(37.9 \%)$ \\
\hline \multicolumn{4}{|l|}{ Potassium } \\
\hline & Normal & $15(93.8 \%)$ & $57(98.3 \%)$ \\
\hline & High & $1(6.3 \%)$ & $1(1.7 \%)$ \\
\hline Duration of complaints (hours) & & $12.9 \pm 5.3$ & $31.3 \pm 16.1$ \\
\hline Duration of hospital stay (days) & & $3.2 \pm 0.8$ & $5.1 \pm 1.6$ \\
\hline Duration of nasogastric tube drainage (days) & & $1.3 \pm 0.5$ & $1.9 \pm 1$ \\
\hline Time of enteral feeding (days) & & $1.7 \pm 0.7$ & $2.5 \pm 1.1$ \\
\hline Duration of radiological recovery (days) & & $2.4 \pm 0.7$ & $4.2 \pm 1.4$ \\
\hline \multicolumn{4}{|l|}{ Surgery } \\
\hline & Yes & 0 & $6(10.3 \%)$ \\
\hline
\end{tabular}




\begin{tabular}{llll}
\hline & & Group 1 $(\mathrm{n}=16)$ (Wild pear) & Group 2 (n=58) (Adhesi \\
\hline & No & $16(100 \%)$ & $52(89.7 \%)$ \\
Follow-up (months) & & $17.2 \pm 2.8$ & $12.5 \pm 4.4$ \\
Recurrence of SBO & Yes & 0 & $11(19 \%)$ \\
& No & $16(100 \%)$ & $47(81 \%)$ \\
\hline
\end{tabular}

Data were reflected as the mean \pm standard deviation except where otherwise indicated.

${ }^{+}$: Student t-test; ${ }^{++}$: Mann-Whitney U test, ${ }^{\text {SS}}$ : Chi-square test, ${ }^{\mathrm{P}}$ : Fisher's exact test.

NA: Not available; SBO: Small bowel obstruction; WBC: White blood cell; CRP: C-reactive protein; BUN: Blood urea nitrogen.

\section{Figure Legends}

Figure 1 a-b: a: Tree and fruit of oleaster-leafed pear (Pyrus elaeagnifolia Pall .). b: Ripened fruits.

Figure 2 a-c: a: Air-fluid levels following wild pear consumption in a patient with a history of operation due to peptic ulcer perforation 30 years ago. b: Dilated small-bowel loops and collapsed ascending colon segment due to wild pear in a patient who had undergone laparoscopic cholecystectomy 11 years ago. Note that wild pear seeds seen in the stomach (arrow). c: Dilated bowel loops in a patient who have no history of abdominal operation or peritonitis. 


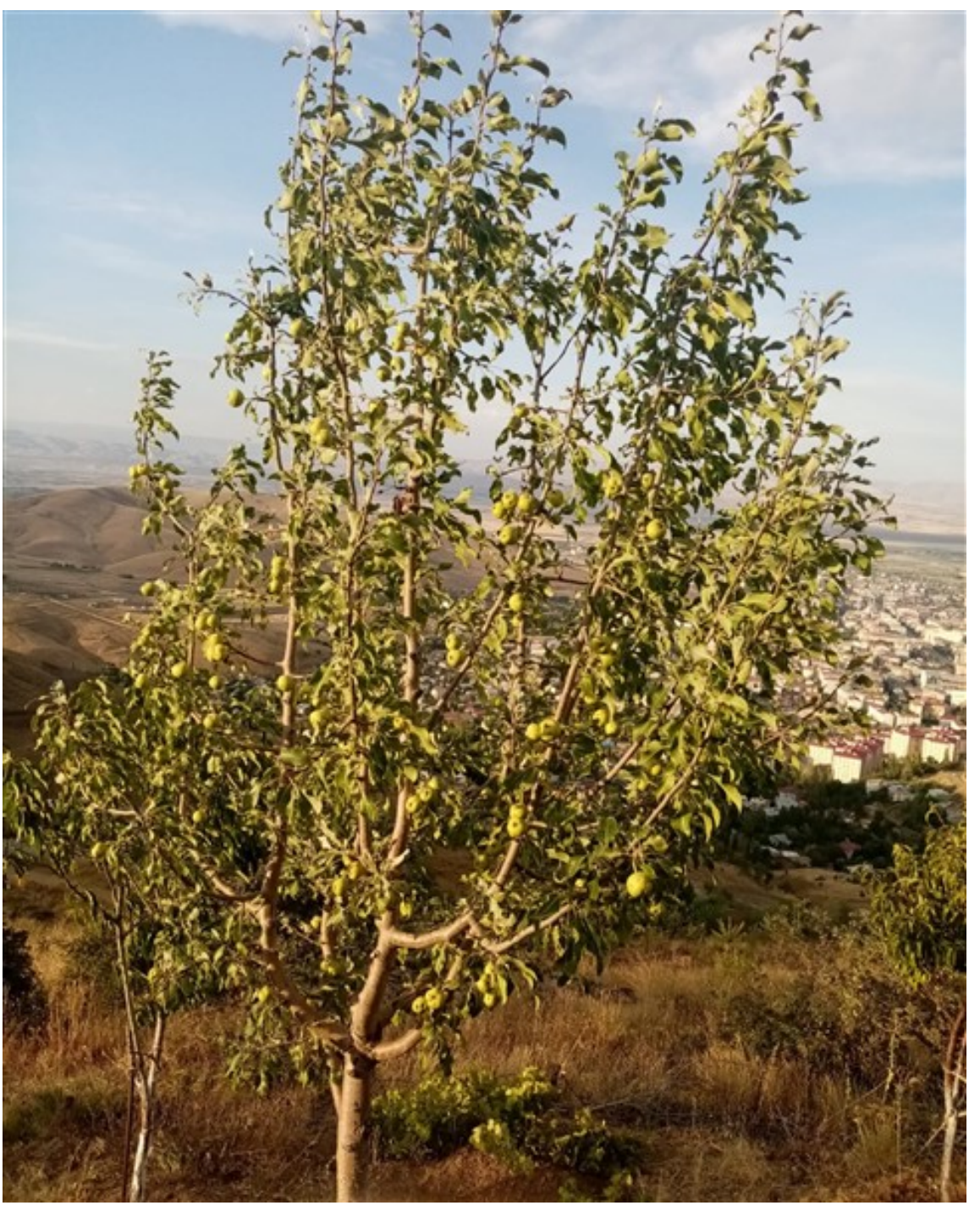




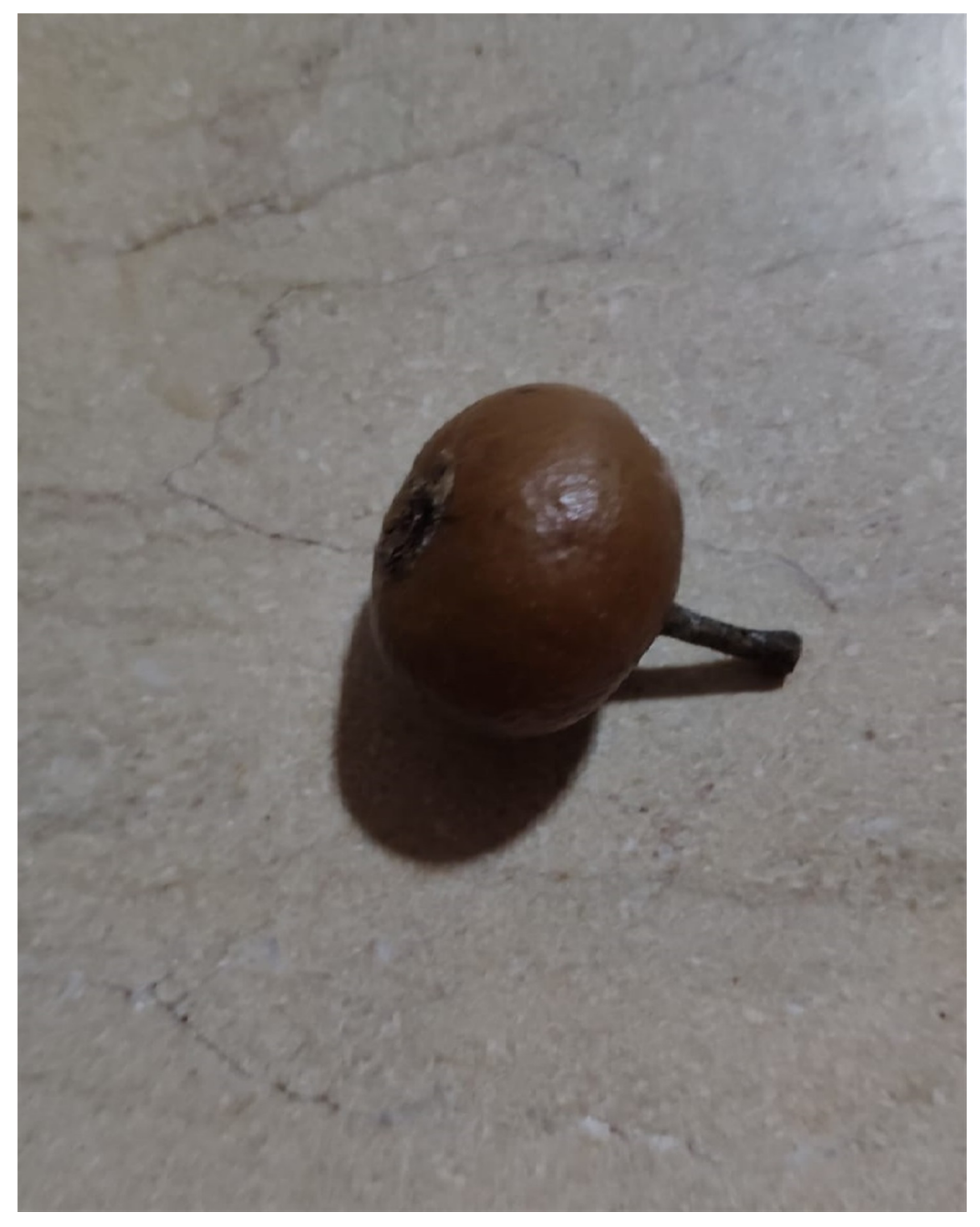



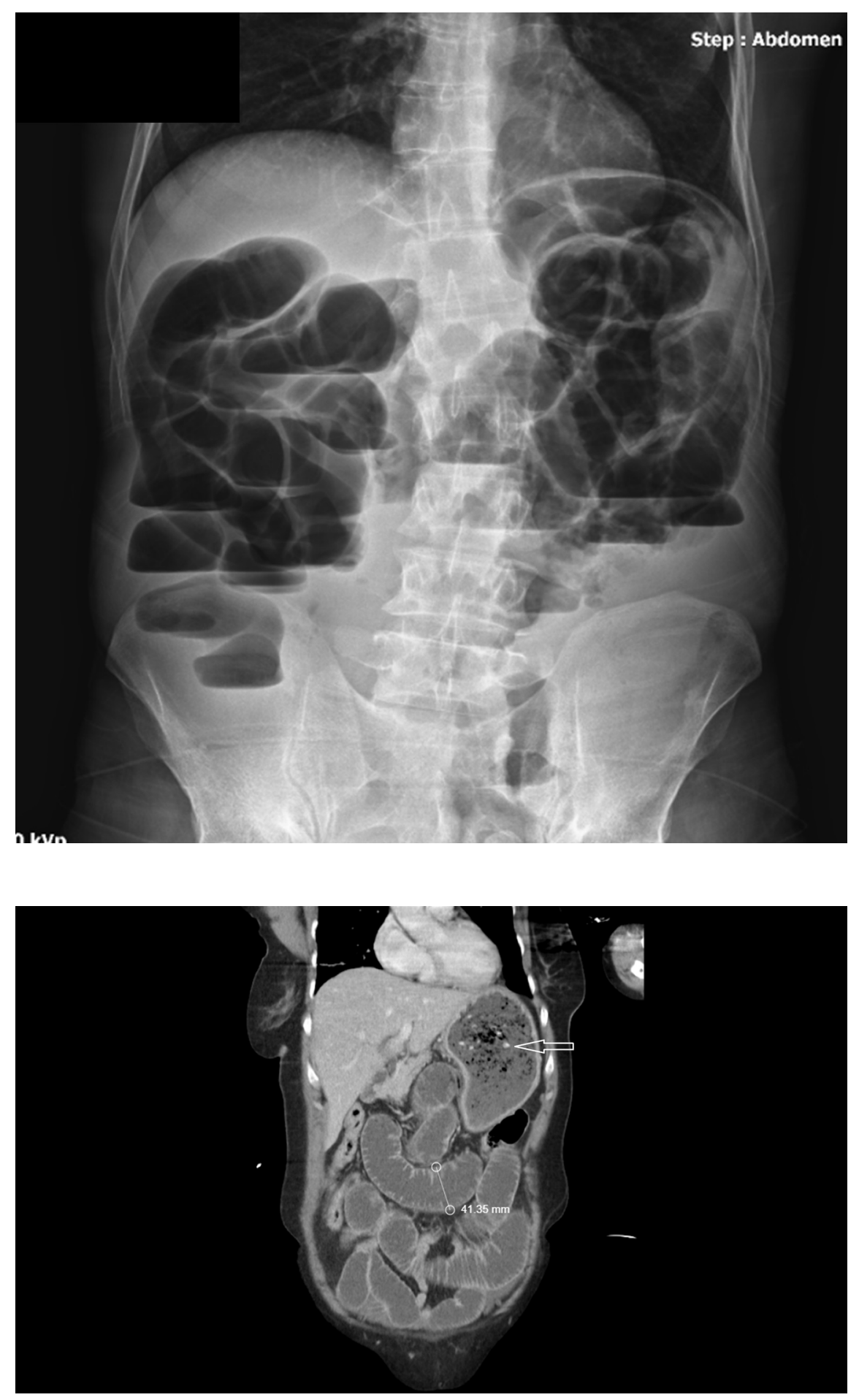


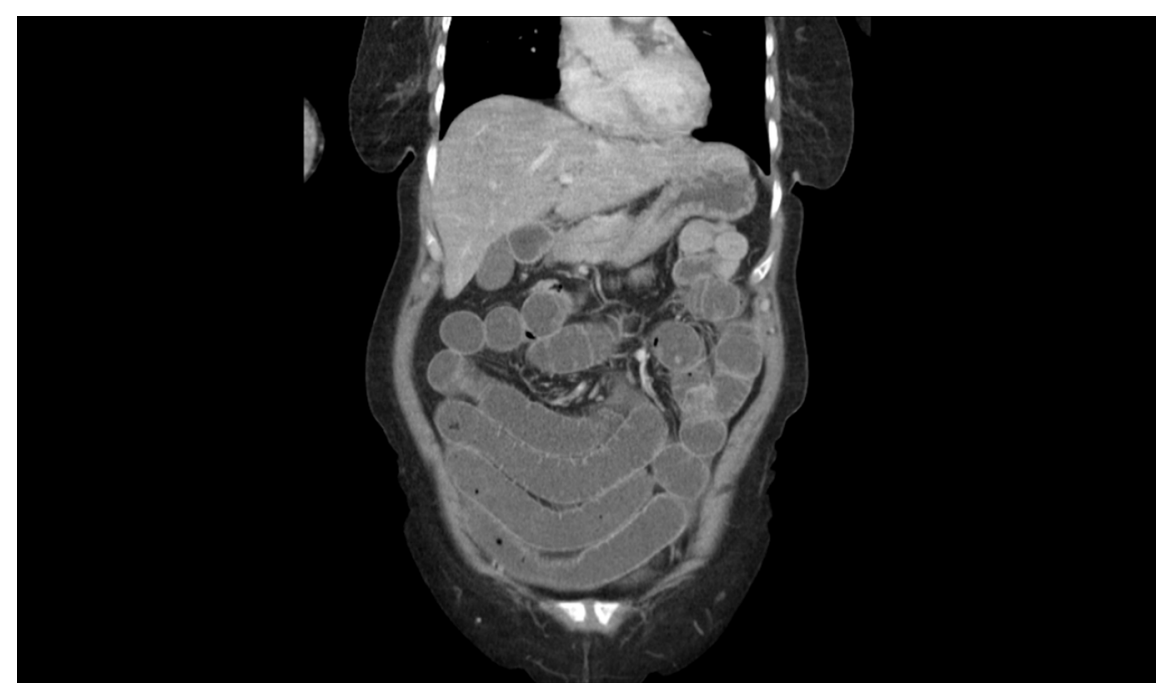

